\title{
Piscicultura marina tropical con peces diádromos: estrategia frente al cambio climático en México
}

\author{
Sergio Escárcega Rodríguez
}

\begin{abstract}
Resumen
Ante la fuerte evidencia del incremento en la intensidad de huracanes y tormentas tropicales derivados del calentamiento global antropogénico (producto de los seres humanos), se prevé que en el mediano y largo plazo los efectos de estos fenómenos se acentúen en los litorales de México. Por lo tanto, resulta imperativo definir escenarios de adaptación para la piscicultura marina que permitan el cultivo en otros ambientes de especies selectas con menor riesgo de afectación. Es aquí donde se destaca la opción del aprovechamiento sostenible de peces diádromos, en particular de los cuasi-catádromos, como los robalos (Centropomidae), que son especies que crecen en agua dulce y desovan en el mar en puntos cercanos a las desembocaduras de los ríos. Su tolerancia a distintos gradientes de salinidad permite, además de su crecimiento, la producción de biomasa a gran escala en el medio marino, salobre y en agua dulce. En este contexto, si se manejan las condiciones requeridas de salinidad en el desove, se podrían cultivar robalos, algunas corvinas (Sciaenidae), jureles (Carangidae) y pargos (Lutjanidae) en agua dulce dentro de espacios protegidos tierra adentro con un menor riesgo de afectación por fenómenos meteorológicos extremos, aparte de que se obtendrían beneficios como un mayor control y menores costos de operación.
\end{abstract}

Palabras clave: Piscicultura marina tropical, calentamiento global, peces diádromos, reconversión productiva, adaptación al cambio climático, Antropoceno.

\section{TROPICAL MARINE FISH FARMING WITH DIADROMOUS FISH. AN ALTERNATIVE TO AD- aptation to climate Change In Mexico}

\begin{abstract}
Given the strong evidence of the increase in the intensity of hurricanes and tropical storms due to anthropogenic global warming, that is, produced by man, it is expected that in the medium and long term this situation will be enhanced on the coastlines of Mexico. It is imperative, therefore, to define adaptation scenarios for marine fish farming that allow the cultivation of selected species in other environments with less risk of affectation. It is here that the option of sustainable use of diadromous fish, particularly quasicatadromes, such as robalos (Centropomidae), which are species that grow in fresh water and spawn in the sea at points close to river mouths. Its tolerance to different salinity gradients allows its growth and the production of large-scale biomass in the marine, brackish, and freshwater environments. In this context, several of them, handling the required conditions of salinity in spawning, could be grown in fresh water, such as robalos, some croaker (Sciaenidae), horse mackerel (Carangidae) and snapper (Lutjanidae), in protected areas, land inside, with a lower risk of affectation due to extreme weather events, lower management and lower operating costs.
\end{abstract}

Keywords: Tropical marine fish farming, global warming, diadromous fish, productive reconversion, adaptation to climate change, Anthropocene.

Recepción: 20/01/2020. Aprobación: 29/07/2020.

Dol: http://doi.org/10.22201/cuaieed.16076079e.2020.21.6.3

Universidad Nacional Autónoma de México, Coordinación de Universidad Abierta, Innovación Educativa y Educación a Distancia (CUAIEED) Este es un artículo de acceso abierto bajo la licencia de Creative Commons 4.0 (c) (i)(2) 


\section{Sergio Escárcega Rodríguez}

Biólogo por la Facultad de Ciencias de la Universidad Nacional Autónoma de México (UNAm) y Maestro en Ciencias en Limnología y Acuicultura por el Instituto de Investigaciones sobre los Recursos Naturales de la Universidad Michoacana de San Nicolás de Hidalgo. Ha sido Jefe de los Centros Acuícolas de Tiacaque, Mex., y de El Peaje, S.L.P., de la Secretaría de Pesca/ SEMARnaP, y Subdelegado de Pesca de la semarnap en los estados de Guanajuato y Michoacán, así como Subdirector de Fomento Pesquero y Acuícola de la Comisión de Pesca y Director de Contención del Deterioro Ambiental de la Secretaría de Urbanismo y Medio Ambiente del gobierno del estado de Michoacán de Ocampo. Ha publicado artículos en materia de acuicultura y cambio climático, en memorias de congresos nacionales e internacionales y artículos en revistas indizadas y arbitradas; además de libros en materia de cultivo de peces. Ha sido comisionado por el gobierno de México en eventos de intercambio técnico y capacitación en materia acuícola en la República Popular China y en Puerto Montt, Chile. Actualmente se desempeña como Consultor independiente. 


\section{Piscicultura marina en México y escenarios futuros}

El incremento en la oferta de alimentos para consumo humano en nuestro país es una necesidad impostergable en virtud de las proyecciones del crecimiento poblacional y de los imperativos que suponen la generación de riqueza, la creación de nuevas fuentes de empleo, el desarrollo regional y la conservación del capital natural de los mares de México.

Un nuevo derrotero en la producción acuícola en el país podría darse con el desarrollo y expansión de la piscicultura marina tropical basada en las especies eurihalinas ${ }^{1}$ de litorales mexicanos debido a su alto valor comercial, vasto potencial biológico (pueden producir enormes cantidades de larvas y crías) y versatilidad para su cultivo. Estas características posibilitan la producción de biomasa a gran escala con fines alimentarios y comerciales en distintos ambientes.

Uno de los factores importantes para asegurar la viabilidad de los proyectos acuícolas en sistemas suspendidos en el mar, es el de disponer de espacios protegidos de fuertes corrientes y oleaje que también faciliten el manejo y aseguramiento de la infraestructura productiva. En el caso de México, los litorales en su franja tropical en las vertientes del Océano Pacífico, Golfo de México y Mar Caribe constituyen zonas de trayectoria frecuente de huracanes durante el verano (Figura 1).

Figura 1. Formación de huracanes en la vertiente del Pacífico de México. Imagen tomada de: https://traficozmg. com/2018/05/comienzatemporada-de-huracanes-enmexico/

1 Peces que toleran amplias variaciones de salinidad en el agua (como los diádromos); tales como los robalos y las corvinas, como casos representativos.

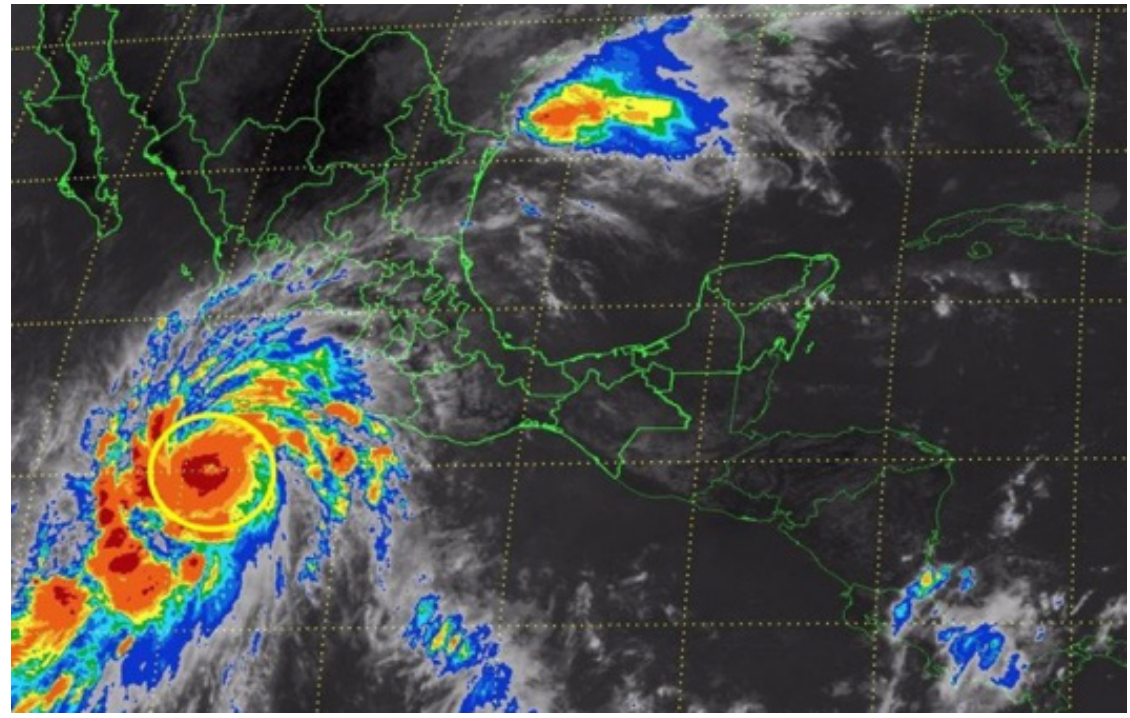

Ante el contundente incremento en la intensidad de estos fenómenos por el calentamiento global, es de esperarse que a mediano y largo plazo esta emergencia se agudice. Por ello, es necesario definir escenarios de adaptación a estos cambios; y la piscicultura marina tropical en México no debe ser la excepción.

A partir de estudios de factibilidad, es posible identificar y referenciar con el mayor grado de certidumbre zonas marinas propicias para el establecimiento 
Figura 2. Cultivo de peces en jaulas flotantes en presas. Imagen tomada de: https:// panoramaacuicola.com/wpcontent/uploads/2019/03/ CUIDAMOS-Malpaso-47.jpg

2 Estos peces en distintas etapas de su ciclo de vida se adaptan a distintos gradientes de salinidad en el agua, pues habitan temporalmente en las partes bajas de los ríos, en las desembocaduras, lagunas costeras y en el ambiente marino (Pérez, 2018).

${ }^{3}$ Ambientes de agua dulce, en este caso los ríos. de sistemas de engorda de peces en jaulas flotantes en las áreas con el menor riesgo de impacto por estos fenómenos. Además, porque habrá que considerar la normatividad vigente en materia de navegación, ordenamiento ecológico territorial y protección ambiental que le den la mayor infalibilidad al desarrollo de la actividad, se hace necesario perfilar opciones para el aprovechamiento de peces marinos en otros ambientes con un menor peligro de afectación.

Esta propuesta tiene por cometido presentar las ventajas que reúnen los peces diádromos tropicales ante el cambio climático global, así como las estrategias y acciones tangibles que se podrán implementar para el desarrollo y expansión de su cultivo en México.

\section{¿Por qué pensar en peces diádromos?}

Es aquí en donde destaca la alternativa del aprovechamiento sostenible en el ciclo completo de especies de peces diádromos² de los litorales de México dentro de su franja tropical. En particular hablamos de los cuasi-catádromos, como los robalos (Centropomidae), que son especies que crecen en agua dulce y desovan en el mar en zonas cercanas a las desembocaduras de los ríos (Cotto et al., 2010).

Su tolerancia a fluctuaciones en la salinidad permite su crecimiento en el medio marino, en el estuarino (desembocaduras de los ríos, lagunas costeras) y en el medio limnético. ${ }^{3}$ En este contexto, si se manejan las condiciones requeridas de salinidad en el desove, se podrían cultivar robalos (Escárcega, 2010), algunas corvinas (Sciaenidae) (FAO, 2005-2018), jureles (Carangidae) (Allen et al., 2002) y pargos (Lutjanidae) (Smith, 1997) en agua dulce dentro de espacios protegidos tierra adentro con un menor riesgo de afectación en la infraestructura por fenómenos meteorológicos extremos, aparte de que se obtendrían beneficios como un un mejor control sobre los cultivos y menores costos de operación (Figura 2).

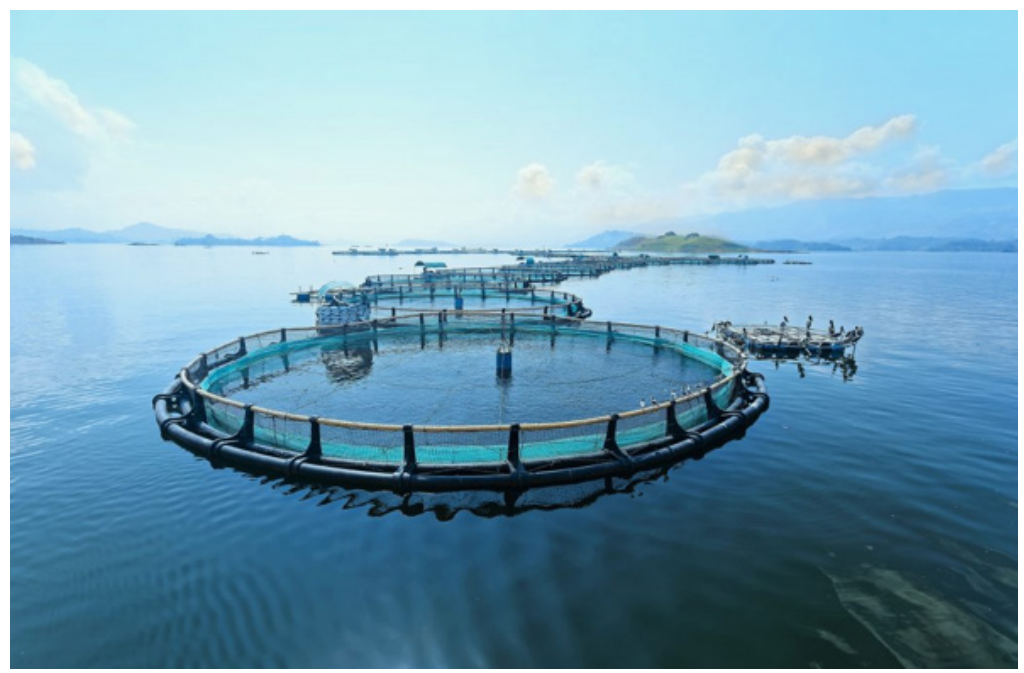


Figura 3. Pacífico Transicional Mexicano (Conabio, 2020). Imagen tomada de: https://www. biodiversidad.gob.mx/region/ ecorregiones-marinas

Figura 4. Ejemplos de peces diádromos con mayor potencial de cultivo en ambientes salobres y de agua dulce en el Pacífico Oriental. Tomado de: EscárcegaRodríguez (2018).

${ }^{4}$ Región que es prácticamente coincidente con la Ecorregión marina del Pacífico transicional mexicano (CONABIO, 2008).

${ }^{5}$ Es la incorporación de juveniles a una pesquería susceptibles de ser capturados.

\section{Las especies selectas}

Por ejemplo, para el caso de región del Pacífico Sur de México, ${ }^{4}$ que abarca los estados costeros de Jalisco a Chiapas (Figura 3), se realizó una preselección de especies eurihalinas de componente marino sobre tres precondiciones básicas: valor comercial, presencia de las mismas en sistemas lagunares y estuarinos, y talla (Escárcega-Rodríguez, 2018).

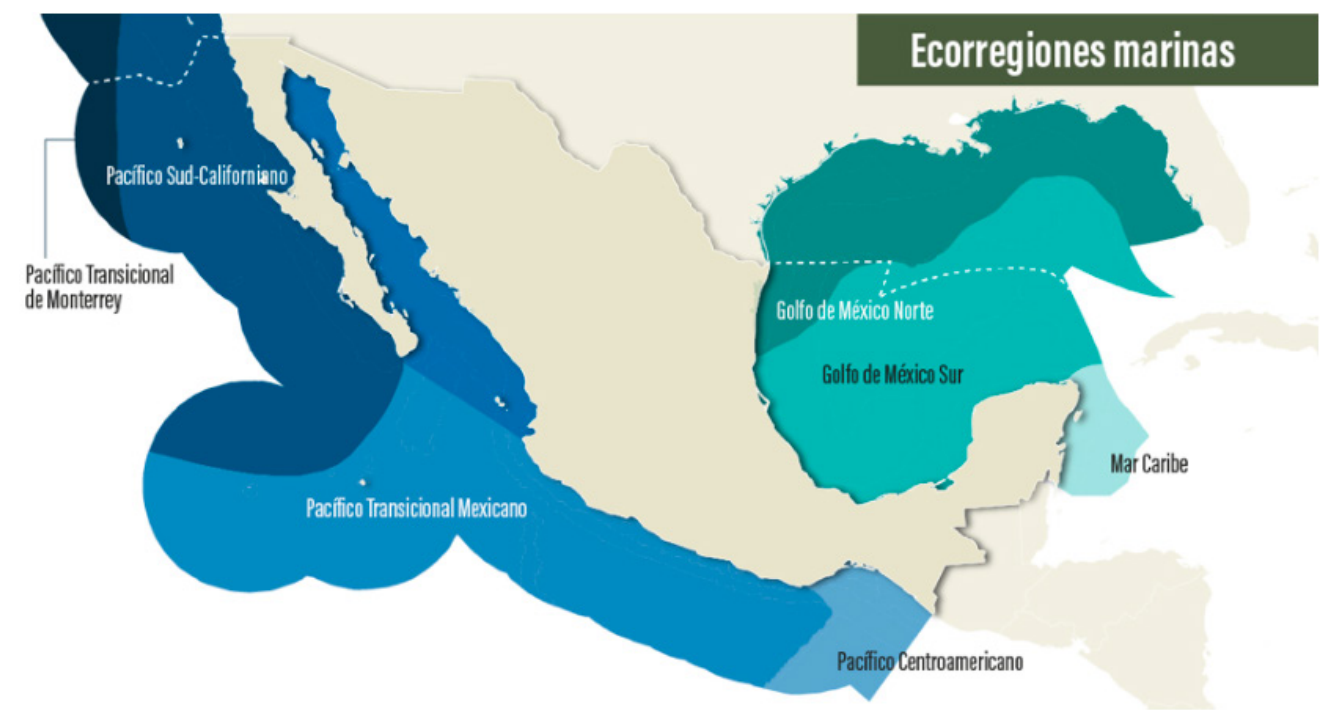

Como resultado de dicho estudio, los puntajes más altos pertenecen a los robalos prieto y plateado (Centropomus nigrescens y $C$. viridis, respectivamente), el mero guasa (Epinephelus itajara), los pargos (como Lutjanus peru y L. colorado), el pámpano (Trachinotus kennedyi) y la corvina (Cynoscion albus) (Figura 4). Se trata de especies selectas consideradas extraordinarias por su alto valor comercial y atributos biológicos para la producción de biomasa a escala masiva. Además, otra importante ventaja en cuanto a la conservación del capital natural de los mares de México y de la región oceánica del Pacífico Oriental Tropical (POT), es que una parte de la producción controlada de crías podría destinarse también para fortalecer el reclutamiento ${ }^{5}$ en las poblaciones de estas especies en su hábitat natural.

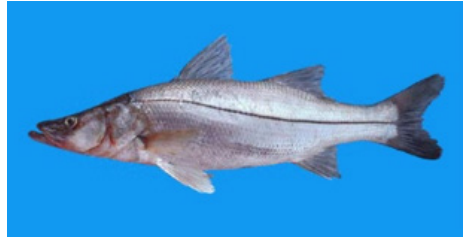

Robalo plateado Centropomus viridis En: FishBase.org

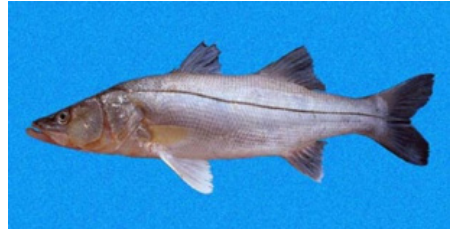

Robalo prieto Centropomus nigrescens En: FishBase.org

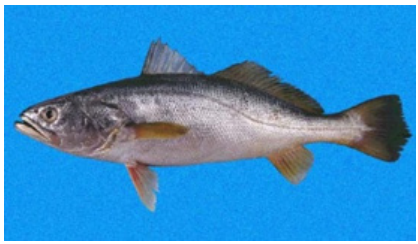

Corvina blanca Cynoscion albus En: FishBase.org 
"Piscicultura marina tropical con peces diádromos: estrategia frente al cambio climático en México"

Sergio Escárcega Rodríguez

Vol. 21, Núm. 6, noviembre-diciembre 2020

Revista Digital Universitaria

\section{La reconversión productiva como estrategia de adaptación}

En el caso de México, y paralelo a los aprovechamientos en el ambiente marino que se puedan implementar a futuro en zonas protegidas de fuertes oleajes, se aprecian dos grandes vertientes en el marco de la reconversión productiva para poner en funcionamiento esta estrategia acuícola de adaptación en respuesta a la exacerbación de huracanes y tormentas tropicales.

La primera se trata de promover el cultivo de especies selectas en las numerosas Unidades de Producción Acuícola (UPA) ${ }^{6}$ que operan en la actualidad tierra adentro (en agua dulce y agua salobre) en las planicies costeras del país a elevaciones menores a los 500 msnm. Estas unidades se dedican principalmente a la producción de tilapia (Oreochromis spp.), bagre de canal (Ictalurus punctatus) e incluso de camarón (Penaeus vannamei), realizando una reconversión hacia la engorda de especies, como los robalos (Centropomus spp.), que son de alto potencial acuícola y mayor valor en el mercado. También se cuenta con la opción de incorporar en estanques a los robalos en la engorda de tilapia para el control de su reproducción, ya que así se aporta un valor agregado a los cultivos.

La segunda de estas vertientes, es sobre la disponibilidad de un importante número de presas de agua en México. La generación de electricidad en una presa es independiente de su elevación. No así el cultivo de las especies mencionadas, que si requieren de altitudes menores a los $500 \mathrm{msnm}$; sobre to do en las vertientes del Océano Pacífico y Golfo de México. ${ }^{7}$ Estas construcciones abren espacios de gran dimensión a manera de espejos de agua y son propicios para el cultivo de peces diádromos en agua dulce. Estas prácticas traen consigo beneficios tanto para su manejo intensivo en jaulas flotantes con altos rendimientos productivos, así como a nivel extensivo por medio de resiembras periódicas de crías, y esto, a su vez, brinda un mayor valor a las pesquerías establecidas. En este sentido, se tendrían dos ventajas adicionales: la captura de tilapias de mayor talla y aprecio comercial como resultado del control de su reclutamiento natural con un depredador; y la generación de mayor derrama económica a través de la pesca deportiva con especies de alto aprecio en este rubro como los robalos. ${ }^{8}$ Todo lo anterior deriva en una alternativa de alto beneficio socioeconómico potencial para distintas regiones del país (Figura 5).

\section{Opciones para el abastecimiento de las crías}

(Pterygoplichthys pardalis) está causando serias afectaciones.

Al respecto, existe evidencia

de que el robalo prieto del Golfo de México (Centropomus poeyi) depreda a dicha especie amazónica (Wakida-Kusunoki et al., 2016), misma que tiene presencia en distintas cuencas hidrográficas de México.

${ }^{9}$ Salida del embrión a partir de la ruptura del corion del huevo.

En cuanto al aprovisionamiento de crías para los sistemas de engorda, se cuenta con opciones tecnológicas que se podrán utilizar con peces marinos en los momentos de desove, fecundación y eclosión. ${ }^{9}$ Se trata de los sistemas acoplados de desove e incubación para especies de huevo pelágico (flotante) de alta fecundidad, como las especies que nos ocupan, en los que se logra la cosecha automática del huevo y la producción masiva de larvas en estadio de alimentación (Escárcega-Rodríguez, 1996). 
Figura 5. Los robalos (Centropomus spp.) constituyen especies de gran atractivo en la pesca deportiva. En: https://i. ytimg.com/vi/g|AVLCWDIv4/ maxresdefault.jpg

\footnotetext{
${ }^{10}$ En el caso de los estanques, es la gradación que se va dando en el tiempo de los principales componentes del zooplancton. Esos pequeños animalitos que flotan en la superficie del agua, como los rotíferos, cladóceros y copépodos, que sirven de alimento a las larvas de los peces.

${ }^{11}$ Los detalles de un concepto de este tipo (reproducción y crianza de peces diádromos) se podrán consultar en: Escárcega
} (2020b).

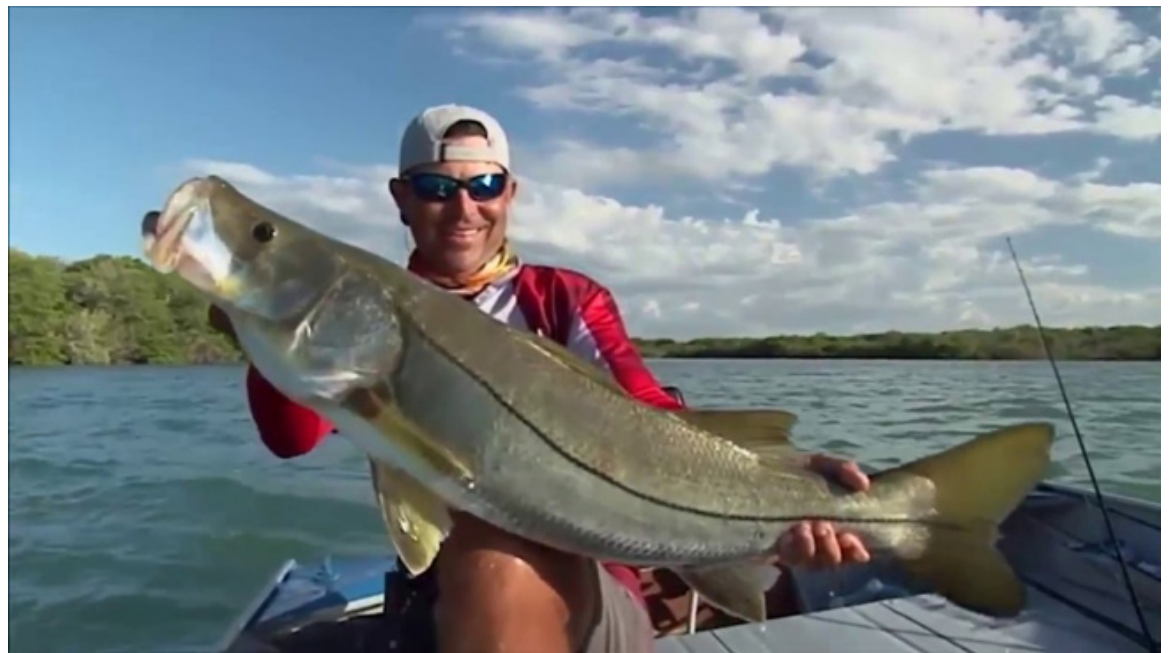

De igual manera, en cuanto a la etapa larval, con base en los resultados favorables logrados en otros países, como por ejemplo con el robalo del IndoPacífico (Lates calcarifer), será conveniente probar protocolos alternativos para el cultivo larval y la crianza en estanques rústicos en los que se acopla de manera sincrónica la siembra de larvas en fase de alimentación (aproximadamente 48 horas después de la eclosión) con la sucesión del zooplancton ${ }^{10}$ que se presenta de manera natural en estanques preparados ex profeso (Escárcega-Rodríguez, 2020a). En estos protocolos aplicados en Australia con L. calcarifer se reportan índices de sobrevivencia en la crianza que varían del 20 al 40\% bajo densidades de siembra de 900000 larvas/ha. Esto, con una ventaja adicional de que se presenta un crecimiento más rápido y mayor vitalidad en las mismas con respecto a los resultados de sistemas intensivos de alto control en recirculación en pequeños contenedores (Rutledge y Rimmer, 1991; Barlow et al., 1996).

En una fase inicial, bajo la batuta del gobierno federal (CONAPESCA), una Unidad demostrativa de este tipo se podría instalar en la costa en donde se aproveche y adapte infraestructura ya existente. De esta manera, se podrá conformar un capital semilla tecnológico para impulsar esta promisoria vertiente productiva y detonar nuevas y significativas cadenas de valor acuícola en México ${ }^{11}$ como se ha hecho con otras especies.

\section{Perspectivas}

Es factible afirmar que un incremento sustancial en la producción acuícola en México podrá lograrse con el aprovechamiento de esta ventana de oportunidad si se considera la disponibilidad de más de 11 mil kilómetros de litorales (en el Océano Pacífico, Golfo de México y Mar (aribe), de un número importante de presas de gran extensión superficial y baja elevación, y de un elenco considerable de peces eurihalinos y diádromos con altos atributos para su cultivo. En este sentido, es preciso destacar que en México y América Latina (AL) es poco lo 
"Piscicultura marina tropical con peces diádromos: estrategia frente al cambio climático en México"

Sergio Escárcega Rodríguez

Vol. 21, Núm. 6, noviembre-diciembre 2020

Revista Digital Universitaria

que se ha avanzado en materia de cultivo de peces marinos tropicales en ciclo completo y el potencial de desarrollo de esta vertiente productiva se aprecia altamente significativo.

Se considera necesario, por tanto, focalizar la investigación sobre los peces diádromos (iniciando con especies como los robalos y corvinas) que reúnen los más altos atributos acuícolas (Escárcega-Rodríguez, 2018) para optimar la aplicación de los recursos que se canalizan para este propósito y facilitar el desarrollo de los paquetes tecnológicos para su aprovechamiento en ciclo completo. Así es como se puede avanzar en el marco del cambio climático en esta época del Antropoceno con estrategias que apuntan a la diversificación productiva, la seguridad alimentaria y el desarrollo regional sostenible en nuestro país y en la región de América Latina.

\section{Referencias}

Allen, G. R., Midgley, S. H. y Allen, M. (2002). Field guide to the freshwater fishes of Australia. Western Australian Museum.

* Barlow, C., William, K. y Rimmer, M. (1996) Sea bass culture in Australia. INFOFISH International 2(96), 26-33. https://www.researchgate.net/publication/285885249_ Sea_bass_culture_in_Australia

* conabio. (2020a, junio 23). Ecorregiones marinas. Biodiversidad Mexicana. https:// www.biodiversidad.gob.mx/region/ecorregiones-marinas

* conabio. (2008). Capital natural de México.Conocimiento actual de la biodiversidad. (Volumen $n^{\circ}$ I) Comisión Nacional para el Conocimiento y Uso de la Biodiversidad.

* conagua. (2017). Principales presas de México. Comisión Nacional del Agua. http:// sina.conagua.gob.mx/sina/tema.php?tema=presasPrincipales\&ver=reporte

- Cotto, A., Acero, A., Rojas, P. y van der Heiden, A. (2010). Centropomus nigrescens. The IUCN Red List of Threatened Species: e.T178064A7483796. Dol: http://dx.doi. org/10.2305/IUCN.UK.2010-3.RLTS.T178064A7483796.en.

* Escárcega-Rodríguez, S. (1996). Evaluación de un sistema acoplado de desove e incubación para la reproducción controlada de la carpa herbívora (Ctenopharyngodon idellus). Ciencia Pesquera. (13), 87-93. https://www.inapesca. gob.mx/portal/documentos/publicaciones/cienciapesquera/CP13/cp13-13.pdf

* Escárcega-Rodríguez, S. (2018). Primeras pruebas de adaptación al cultivo extensivo del robalo (Centropomus nigrescens) en estanques en la costa de Michoacán. CIENCIA ergo-sum, 25(3). Dol: http://doi.org/10.30878/ces.V25n3a8

* Escárcega-Rodríguez, S. (2018). Preselección de especies para la piscicultura marina en el Pacífico Sur de México. cIENcIA ergo-sum, 25(1). https://cienciaergosum. uaemex.mx/article/view/9230 
* Escárcega-Rodríguez, S. (2020a). ¿Cultivo de peces marinos? Hablemos de larvicultura en estanques. Revista Digital Universitaria, 21(2). Dol: http://doi. org/10.22201/codeic.16076079e.2020.v21n2.a3

* Escárcega-Rodríguez, S. (2020b). Catálogo de especies para la piscicultura marina en el Pacífico Sur de México. AGT Editor.

* FaO. (s. f.). Programa de información de especies acuáticas Sciaenops ocellatus. Departamento de Pesca y Acuicultura de la Fao. Consultado el 27 Julio 2020. http:// www.fao.org/fishery/culturedspecies/Sciaenops_ocellatus/es

* Pérez, J.I. (2018). Regulación osmótica de los peces diádromos. Animalia. Cuaderno de cultura científica. Consultado el 27 Julio 2020. https://culturacientifica. com/2018/05/08/la-regulacion-osmotica-de-los-peces-diadromos

* Rutledge, W.P. y Rimmer, M.A. (1991). Culture of larval sea bass, Lates calcarifer (Bloch), in saltwater rearing ponds in Queensland, Australia. Asian Fisheries Society, 4(3), 345-355. https://bit.ly/3dOVyQg

* Smith, C.L. (1997). National Audubon Society field guide to tropical marine fishes of the Caribbean, the Gulf of Mexico, Florida, the Bahamas, and Bermuda. Alfred A. Knopf, Inc.

Wakida-Kusunoki, A.T. y Toro-Ramírez, A. (2016). El robalo prieto (Centropomus poeyi), nuevo depredador del pez diablo (Pterygoplichthys pardalis). Hidrobiológica 26(1), 147-149. http://www.scielo.org.mx/scielo.php?script=sci_arttext\&pid $=$ S0188-88972016000100147

\section{Cómo CITAR ESTE ARTículo}

* Escárcega Rodríguez, Sergio. (2020, noviembre-diciembre). Piscicultura marina tropical con peces diádromos: estrategia frente al cambio climático en México. Revista Digital Universitaria (RDU), 21(6). Dol: http://doi.org/10.22201/ cuaieed.16076079e.2020.21.6.3 